\title{
II. Prevalence of peripheral arthritis, sacroiliitis, and ankylosing spondylitis in patients suffering from inflammatory bowel disease
}

\author{
BETTY J. DEKKER-SAEYS, S. G. M. MEUWISSEN, ELLA M. VAN DEN \\ BERG - LOONEN, W. H. D. DE HAAS, D. AGENANT, AND G. N. J. TYTGAT
}

From the Amsterdam Centre for Rheumatic Diseases, the Division of Gastroenterology, Department of Medicine, University of Amsterdam, and the Central Laboratory of the Netherlands Red Cross Blood Transfusion Service, Amsterdam

SUMmARY To establish the prevalence of peripheral arthritis, radiographic sacroiliitis, and ankylosing spondylitis in patients with inflammatory bowel disease, 58 consecutive patients suffering from ulcerative colitis (UC) and 51 with Crohn's disease (CD) underwent a detailed rheumatological examination. In addition, all patients were screened for the presence of the antigen HLA B27. Peripheral arthritis was found in 14 (8 UC, $6 \mathrm{CD})$ patients $(12 \cdot 8 \%)$; radiographic sacroiliitis was diagnosed in 11 (5 UC, $6 \mathrm{CD})(10 \cdot 1 \%)$, of whom 10 were asymptomatic; and ankylosing spondylitis was diagnosed in 2 UC and 2 CD patients (3.7\%). $18.9 \%$ of the UC and $3.9 \%$ of the CD patients were HLA B27 positive. One of the 11 patients with radiographic sacroiliitis and 2 of the 4 with ankylosing spondylitis had the HLA B27 antigen.

Peripheral arthritis, radiographic sacroiliitis, and ankylosing spondylitis are apparently frequent manifestations in patients suffering from inflammatory bowel disease. Asymptomatic radiographic sacroiliitis in these patients appears to differ from idiopathic ankylosing spondylitis, both clinically and genetically. Evaluation of subjective rheumatological complaints, necessary for a confident diagnosis of ankylosing spondylitis, according to the New York criteria is difficult during a flare-up of the inflammatory bowel process, as was shown in $4 \mathrm{CD}$ cases with marked limitation of lumbovertebral function and chest expansion, but no radiological abnormalities of the SI joints.

The occurrence of joint abnormalities is a well-known association of idiopathic inflammatory bowel disease. Peripheral arthritis may occur with joint activity running more or less parallel with the inflammatory bowel disease and should not be confused with rheumatoid arthritis. On the other hand, a central form consists of radiographic sacroiliitis (RSI) and ankylosing spondylitis (AS). The clinical course of the latter two running more or less independently of the bowel disease (Ford and Vallis, 1959; Acheson, 1960; McEwen et al., 1962; Rotstein et al., 1963; McBride et al., 1963; Wright and Watkinson, 1965). The reported incidence of peripheral arthritis varies from $2 \cdot 2 \%$ (Crohn and Yarnis, 1958) to $22 \cdot 2 \%$ (Hammer et al., 1968). The incidence of RSI varies from $4.1 \%$ (Jacoby and Jayson, 1974) to $19.8 \%$ (Ansell and Wrighley, 1964), and of AS from 3\% (Acheson, 1960) to $7 \cdot 0 \%$ (Moll et al., 1974).

Accepted for publication July 18, 1977

Correspondence to Professor Dr G. N. J. Tytgat, Division of Gastroenterology, Wilhelmina Gasthuis, 1 e Helmersstraat 104, Amsterdam, The Netherlands.
The purpose of the present study was to establish the prevalence of RSI and AS in a large group of nonselected consecutive patients, suffering from ulcerative colitis (UC) and Crohn's disease (CD). In addition, these patients were typed for the histocompatibility antigen HLA B27 because of the well-known strong association between HLA B27 and AS (Brewerton et al., 1973; Schlosstein et al., 1973; Amor et al., 1974; van den Berg-Loonen et al., 1977).

\section{Patients and methods}

Fifty-eight nonselected UC and 51 CD patients, attending the Division of Gastroenterology, were asked to co-operate in the study. Diagnosis of inflammatory bowel disease (UC and CD) was made according to accepted clinical, endoscopic, radiological, and histological criteria, or was confirmed at surgery, in agreement with criteria described by Schachter and Kirsner (1975). A detailed rheumatological analysis of all patients with bowel disease was made by one of us (B.D.S.). In addition, $x$-ray 
studies of the SI joints, if necessary combined with $x$-rays of the lumbar spine, were made using a standard technique. All radiographs were examined by two independent experienced radiologists without prior knowledge of the clinical findings. The results were graded according to Bennett and Burch (1967) as $0=$ normal SI joint, $1=$ suspicious RSI, 2= abnormal SI joint with sclerosis and/or erosions, $3=$ unequivocally abnormal with erosions, sclerosis, widening or narrowing or partly ankylosed, and $4=$ total ankylosis. The spine was examined for syndesmophytes and/or ligamentous calcification. The diagnosis of definite or probable AS was made according to the New York criteria (Bennett and Burch, 1967).

Blood was taken for histocompatibility testing by a modified NIH microlymphocytotoxicity technique (Engelfriet et al., 1972). The detailed results of HLA typing of 33 antigens of the A, B, and C loci in these $\mathrm{UC}$ and $\mathrm{CD}$ patients have been reported elsewhere (van den Berg-Loonen et al., 1977). This study was limited to the HLA B27 antigen.

\section{Results}

Fifty-eight UC patients (mean age $42 \cdot 1$ years, range 19-77 years) were studied, 24 males and 34 females (mean ages 34.3 and 44.7 years respectively). 51 CD patients (mean age 37.2 years, range 17-63 years) were studied, 21 males and 30 females (mean ages 35.5 and 38.4 years respectively). Mean age of onset of inflammatory bowel disease was 30.3 years for the UC patients and 26.7 years for the CD patients. $11 \mathrm{UC}$ patients $(18.9 \%)$ and 2 CD patients $(3.9 \%)$ were HLA B27 positive.

Relevant details of the history, clinical examination, and radiological findings are summarised in the Table. Low back pain was encountered most frequently, its intensity often running in parallel with the activity of the bowel disease, particularly in CD. Peripheral joint involvement consisted of a monoor oligoarthritis, often symmetrical and occurring mainly in the legs. There was no obvious correlation between the presence of HLA B27 and a positive family history of AS or bowel disease, low back pain, or a positive history of peripheral arthritis or iridocyclitis.

Eleven of the 58 UC patients $(18.9 \%)$ and 10 of the $51 \mathrm{CD}$ patients $(19.6 \%)$ showed abnormal SI joints. Of these 21 cases, 6 were classified as suspicious sacroiliitis (RSI grade 1). RSI grade 2 or 3 was diagnosed in 12 (3 HLA B27 positive) and 3 patients (1 HLA B27 positive) respectively, as shown in the Table. Only 4 of these 15 patients with RSI grade 2 or 3 fulfilled the New York criteria for definite AS (2 UC, 2 CD patients); 2 AS cases were HLA B27
Table History and clinical, radiological, and histocompatibility data (HLA B27) in patients suffering from ulcerative colitis and Crohn's disease (numbers of HLA B27 positive patients in parentheses)

\begin{tabular}{lll}
\hline & $\begin{array}{l}\text { Ulcerative } \\
\text { colitis }(n)\end{array}$ & $\begin{array}{l}\text { Crohn's } \\
\text { disease }(n)\end{array}$ \\
\hline Males & $24(6)$ & $21(1$ \\
Females & $34(5)$ & $30(1$
\end{tabular}

History

Positive family history

AS

IBD

Low back pain

in relation to bowel activity

Peripheral arthritis

in relation to bowel activity

Erythema nodosum

Iridocyclitis

\begin{tabular}{|c|c|}
\hline 1 (1) & - \\
\hline $6(3)$ & 7 (1) \\
\hline 17 (4) & $20(1)$ \\
\hline 4 & 12 \\
\hline $8(3)$ & $6(1)$ \\
\hline 5 & 4 \\
\hline 21 & $\overline{3}(1)$ \\
\hline
\end{tabular}

Clinical examination

Symptoms of arthritis

Limited spinal function

Limited spinal function and/or reduced chest expansion

4 (1) $\quad 2(-)$

$2(-)$

$-$

$3(1)$

$5(-)$

Radiological examination

Radiographic sacroiliitis grade 1 (suspicious)

2

2

4

Lumbar spine involvement

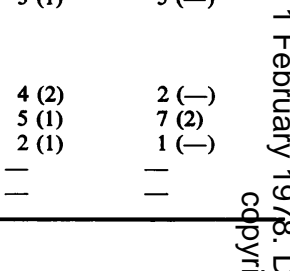

positive (1 UC, $1 \mathrm{CD}) .10$ of the other 11 patie with grade 2 or 3 were completely asymptomatic (主 females, 4 males). In none of the AS or RSI patient was there radiological evidence of lumbar spin 8 involvement. No correlation was found between the severity of RSI and duration of bowel disease, sit $\overrightarrow{\vec{E}}$ of disease, or extent of gut involvement.

Four CD patients had severe low back pain marked limitation of lumbar spine mobility, an reduced chest expansion when first seen. Because of these impressive features and the physical findings, 8 diagnosis of AS was seriously considered. However radiological examination of the SI joints and spine showed no abnormalities. During the follow-up study it became evident that the rheumatologica complaints and clinical abnormalities were running in parallel with the bowel disease. In all 4 patients internal or external fistulae were present at the start of the study, or had been present previously All 4 were HLA B27 negative.

\section{Discussion}

In this survey of a group of nonselected patients wit inflammatory bowel disease peripheral arthriti occurred in $12.8 \%$, RSI in $10.1 \%$, and AS in $3.7 \%$ The frequencies of these complication in UC ans CD were similar. 
Peripheral arthritis was usually monarticular, occasionally oligoarticular, mainly affecting the legs and often running in parallel with intestinal inflammatory activity, as extensively described in previous studies (Wright and Watkinson, 1965; Palumbo et al., 1973; Deshayes et al., 1976a, b). 5 UC and 6 CD patients had an RSI grade of 2 or $3(10.1 \%)$, a frequency comparable with the figures of Ansell and Wrighley (1964) of $12 \%$, Wright and Watkinson (1965) 18\%, Mueller et al. (1974) 7\%, Moll et al. (1974) $11.2 \%$, Mallas et al. (1976) UC 6\% and CD $11 \%$, and Hyla et al. (1976) $12.4 \%$. 7 out of 11 RSI patients in the latter study were females, with no further clinical evidence of AS; only 1 was HLA B27 positive $(9.1 \%)$. We noticed a similar trend: female preponderance (7 of 11 cases), asymptomatic course of disease (10 of 11), and a low frequency of HLA B27. Such features are highly unusual for idiopathic AS, and we therefore agree with Hyla et al. that asymptomatic RSI is not likely to be a precursor of idiopathic AS.

Only 4 of the 109 patients with bowel disease fulfilled the New York criteria for the diagnosis of AS $(3.7 \%)$, corresponding to the low frequency found by Wright and Watkinson (1965) of $6.4 \%$, Haslock and Wright (1973) 6.9\%, Moll et al. (1974) $7.0 \%$, Mueller et al. (1975) 3.7\%, Hyla et al. (1976) $4.5 \%$, and Greenstein et al. (1976) $4 \% .2$ of our 4 patients were HLA B27 positive; both had other signs of idiopathic AS: a positive family history of AS in 1, and in both a history of iridocyclitis and peripheral arthritis not related to activity of inflammatory bowel disease. In the 2 HLA B27 negative AS patients these features were lacking.

Eleven of the $58 \mathrm{UC}$ and 2 of the $51 \mathrm{CD}$ patients were HLA B27 positive. Van den Berg-Loonen et al (1977) recently analysed the histocompatibility data of these two patient groups in detail. No statistical differences were shown between UC or CD patients and healthy controls, with regard to the presence of HLA B27, although evidence is now available from a combined study at Liverpool, London, and Amsterdam that the frequency of B27 is increased in UC but not in CD (J. C. Woodrow et al., unpublished). A similar trend has been observed by Mallas et al. (1976). It should however be emphasised that those UC or CD patients in our study with the HLA B27 antigen manifested an increased frequency of associated rheumatological abnormalities. Indeed 4 of the 11 UC (36.4\%) and both HLA B27 positive CD patients had SI joint pathology. Calin and Fries (1975) and Cohen et al. (1976) reported that healthy individuals carrying the antigen HLA B27 have a higher risk for the development of RSI or AS. A similar conclusion was drawn by Mallas et al. (1976) in $\mathrm{UC}$ and $\mathrm{CD}$ patients.

A diagnosis of AS in patients with inflammatory bowel disease should be based not only on a detailed radiological study of the SI joints and spine, but also on the accurate evaluation of subjective rheumatological complaints in relation to the activity of the intestinal inflammatory process. A striking observation in our series was that 4 HLA B27 negative $C D$ patients suffered from severe low back pain and limitation of spinal mobility and chest expansion, but had no detectable radiological abnormalities. The improvement of rheumatological complaints, in particular of lumbar musculature hypertonia, in conjunction with improved intestinal inflammatory activity might be explained by neurogenic reflexes.

The spectrum of rheumatological abnormalities in patients suffering from inflammatory bowel disease consisted of peripheral arthritis, radiological sacroiliitis - often asymptomatic, with a low frequency of HLA B27-ankylosing spondylitis, and finally a clinical picture suggesting AS, but with no radiological evidence of AS. 